\title{
Toroidal quadrupole transitions associated to collective rotational-vibrational motions of the nucleus
}

[Toroidal quadrupole transitions]

\author{
Ş Mişicu \\ National Institute for Earth-Physics, Bucharest Magurele, P.O.Box Mg-2, ROMANIA
}

\begin{abstract}
In the frame of the collective model one computes the longitudinal, transverse and toroidal multipoles corresponding to the excitations of low-lying levels in the ground state band of several even-even nuclei by inelastic electron scattering $\left(e, e^{\prime}\right)$. In connection with this transitions a new quantity, which accounts for the deviations from the Siegert theorem, is introduced in the frame of the Riemann Rotational Model with non-vanishing vorticity. Inelastic differential cross-sections calculated at backscattering angles shows the dominancy of toroidal form-factors over a broad range of momentum transfer
\end{abstract}

\section{Introduction}

A still opened question in nuclear structure theory concerns the nature of current flows inside the nucleus. Many attempts have been made up to the present time in order to clarify whether the nuclear matter can be portrayed as a quantum fluid that could support only irrotational flows (IF) or is a quantum rotator which give rise to rigid rotation of the whole nucleus (RR) [1]. There have been also proposed intermediate models, like the Riemann rotational model [2] , in which the velocity field is assumed to be a linear combination of rigid and irrotational contributions. Since these models give quite different descriptions for the collective current distributions, a direct determination of the nuclear current is required. The measurement of transverse multipoles in inelastic electron scattering, provides a practical tool for this purpose [3]. The longitudinal (Coulomb) multipole is related to the charge distribution of the nucleus due to the charge-current conservation law. In order to obtain the quantities which depend on the nuclear current one have to measure the transverse part of the cross-section, i.e. the electric and magnetic multipoles.

There is a large amount of information concerning the theoretical calculations and experimental determination of electromagnetic multipoles for such reactions. Our aim is 
to qualitatively investigate the transverse electric multipoles from the point of view of a multipole decomposition introduced by Dubovik and Cheshkov [4]. This decomposition, contains in the second order of momentum transfer the toroidal multipole moment which is independent of the other two classes of known electromagnetic multipoles: the charge and the magnetic one. The first member of this new class of electromagnetic multipoles is called the toroidal dipole or anapole moment, and was introduced by Zel'dovich [5] in connection with the parity violation in weak interactions. The second member is the toroidal quadrupole or tetrapole which is a parity-even and time-odd electromagnetic characteristic [6-8]. In this paper we are interested in the dynamic counterpart, i.e. the induced toroidal moments. Such characteristics turn out to be associated with a isoscalar $\lambda^{\pi}=1^{-}$state, called the dipole torus mode, recently predicted in the frame of nuclear fluid dynamics $[9,10]$. Calculations of the electromagnetic form factors entering into the photoabsorbtion or electron scattering cross sections show that only the toroidal form factor contributes to the excitation of this mode [11]. Therefore for such excitation in the low- $q$ limit we obtain the toroidal dipole transition, not the charge dipole one as is stated by the Siegert theorem for the case of electric GDR.

\section{Toroidal multipoles}

The study of normal-parity excitations in nuclei require the knowledge of longitudinal(Coulomb) and transverse electric multipoles. For simplicity we consider their expressions in Born approximation (PWBA)

$$
\begin{aligned}
& \hat{T}_{\lambda \mu}^{L}=\frac{i^{\lambda+1}}{\sqrt{2 \lambda+1}}\left(\sqrt{\lambda} \delta_{\lambda^{\prime}, \lambda-1}+\sqrt{\lambda+1} \delta_{\lambda^{\prime}, \lambda+1}\right) \int d^{3} r j_{\lambda^{\prime}}(q r) \boldsymbol{Y}_{\lambda \lambda^{\prime}}^{\mu}(\Omega) \cdot \hat{\boldsymbol{J}}(\boldsymbol{r})(1) \\
& \hat{T}_{\lambda \mu}^{E}=\frac{i^{\lambda+1}}{\sqrt{2 \lambda+1}}\left(\sqrt{\lambda+1} \delta_{\lambda^{\prime}, \lambda-1}-\sqrt{\lambda} \delta_{\lambda^{\prime}, \lambda+1}\right) \int d^{3} r j_{\lambda^{\prime}}(q r) \boldsymbol{Y}_{\lambda \lambda^{\prime}}^{\mu}(\Omega) \cdot \hat{\boldsymbol{J}}(\boldsymbol{r})(2)
\end{aligned}
$$

where

$$
\boldsymbol{Y}_{\lambda \lambda^{\prime}}^{\mu}(\Omega)=\left[Y_{\lambda^{\prime}}(\Omega) \otimes \boldsymbol{e}_{1}\right]_{\lambda}^{\mu}
$$

are the vector spherical harmonics [12] and $\hat{\boldsymbol{J}}(\boldsymbol{r})$ is the proton current. Choosing the z-direction to be along the momentum transferred from the electron to the nucleus $\boldsymbol{q}$, the longitudinal and transverse electric reduced matrix elements (r.m.e.) are

$$
\begin{aligned}
t_{L \lambda}(q)_{f i} & \equiv\left\langle I_{f}\left\|\hat{T}_{\lambda}^{L}(q)\right\| I_{i}\right\rangle \\
t_{E \lambda}(q)_{f i} & \equiv\left\langle I_{f}\left\|\hat{T}_{\lambda}^{E}(q)\right\| I_{i}\right\rangle
\end{aligned}
$$


For low momentum transfer, one sees from eq.(1) that Siegert's theorem [13]

$$
t_{E \lambda}(q \rightarrow 0)_{f i}=\sqrt{\frac{\lambda+1}{\lambda}} t_{L \lambda}(q \rightarrow 0)_{f i}
$$

is satisfied, independently of the approximation used for the nuclear current. While this relation is very important for low-energy real-photon processes, it is not suitable at higher momentum transfer. Following the ideas outlined in [4,14], one can build a variant of Siegert's theorem at arbitrary momentum $q$. Thus the r.m.e. of the transverse electric multipole $t_{E \lambda}(q)$ can be separated in two parts: one depending on the low momentum transfer $q$ limit and the other on the high- $q$ terms

$$
t_{E \lambda}(q)_{f i}=\sqrt{\frac{\lambda+1}{\lambda}} t_{L \lambda}(0)_{f i}+q^{2} t_{T \lambda}(q)_{f i}
$$

where $t_{T \lambda}(q)_{f i}$ is the r.m.e. of the toroidal multipole operator

$$
\hat{T}_{\lambda \mu}^{T}=\frac{i^{\lambda}}{q^{3}} \int d^{3} r \boldsymbol{\nabla} \times\left\{\left[j_{\lambda}(q r)-\frac{(q r)^{\lambda}}{(2 \lambda+1) ! !}\right] \boldsymbol{Y}_{\lambda \lambda}^{\mu}(\Omega)\right\} \cdot \hat{\boldsymbol{J}}(\boldsymbol{r})
$$

Therefore $t_{T \lambda}(q)$ contains the whole information of $t_{E \lambda}(q)$ for high- $q$. In the longwavelength limit the toroidal multipole $t_{T \lambda}(0)$ is proportional to the transition toroidal multipole moment $\boldsymbol{T}_{\lambda}$

$$
t_{T \lambda}(0)=-\frac{(i q)^{\lambda-1}}{(2 \lambda+1) ! !} \sqrt{\frac{\lambda+1}{\lambda}} \mathcal{T}_{\lambda}
$$

For $\lambda=1$, we have the earlier mentioned toroidal dipole moment vector operator

$$
\hat{\mathbf{T}}=\frac{1}{10} \int d^{3} r\left[\boldsymbol{r}(\boldsymbol{r} \cdot \hat{\boldsymbol{J}}(\boldsymbol{r}))-2 r^{2} \hat{\boldsymbol{J}}(\boldsymbol{r})\right]
$$

This electromagnetic moment is associated with a poloidal current flow on the wings of a toroidal solenoid. More details can be found in the excellent review [14]. The next terms in the $q$-expansion of the toroidal multipoles are the average 2 n-power radii of the current distribution $\rho_{\lambda \mu}^{2 n}$, which give rise to the corresponding toroidal multipole moment. Their general form is

$$
\hat{\rho}_{\lambda \mu}^{2 n}=\int d^{3} r r^{\lambda+2 n+1}\left(\boldsymbol{Y}_{\lambda \lambda-1}^{\mu}(\Omega)+\frac{2 n+2}{2 \lambda+2 n+1} \sqrt{\frac{\lambda}{\lambda+1}} \boldsymbol{Y}_{\lambda \lambda+1}^{\mu}(\Omega)\right) \cdot \hat{\boldsymbol{J}}(\boldsymbol{r})(
$$

We end this section by noticing that from the point of view of other multipole expansion treatments, the toroidal moments can be viewed as electric multipoles induced by magnetization [15] or as retardation corrections to the Siegert theorem [16].

\section{Deviations from Siegert's theorem in the collective model}

In order to illustrate the ideas presented in the previous chapter we consider the Riemann Rotational Model, which is an algebraic extension of the Bohr-Mottelson model. This 
model assumes that the velocity field is a linear function of the position. A Riemann rotor is characterized by a parameter $\tilde{r}$, called rigidity, which ranges from zero to one. If $\tilde{r}=1$ the body is rotating rigidly (RR) with the angular frequency $\boldsymbol{\omega}$; when $\tilde{r}=0$ the flow is irrotational (IF) and the nuclear surface performs harmonic oscillations with amplitudes given by the collective coordinates $\alpha_{l m}$. The rigidity is related to the quantity called vorticity $\mathcal{L}[17,18]$. The velocity vector field of a Riemann rotor is a convex combination of the rigid and irrotational values,

$$
\hat{\boldsymbol{J}}(\boldsymbol{r})=\tilde{r} \hat{\boldsymbol{J}}_{R R}(\boldsymbol{r})+(1-\tilde{r}) \hat{\boldsymbol{J}}_{I F}(\boldsymbol{r})
$$

where the RR and IF velocity fields are given by

$$
\boldsymbol{v}_{R R}=\boldsymbol{\omega} \times \boldsymbol{r}
$$

and

$$
\left.\boldsymbol{v}_{I F}=R^{2} \sum_{l m} \frac{\dot{\alpha}_{l m}}{l}\left[\nabla\left(\frac{r}{R}\right)^{l}\right] Y_{l m}(\theta, \phi)\right] \theta(R-r)
$$

Introducing the above equations in the expressions of longitudinal and transverse electric multipoles, for an axial-symmetric nucleus with quadrupole deformation $\beta$ one get

$$
\hat{T}_{2 \mu}^{L}(q, \tilde{r})=-Z e \sqrt{\frac{2}{\pi}} \frac{\sqrt{30}}{40} \frac{Q_{0}}{R} \mu \omega_{\mu}\left(j_{1}(q R)+j_{3}(q R)\right)
$$

and

$$
\hat{T}_{2 \mu}^{E}(q, \tilde{r})=-Z e \sqrt{\frac{3}{\pi}} \frac{\sqrt{30}}{40} \frac{Q_{0}}{R} \mu \omega_{\mu}\left(j_{1}(q R)+\left(1-\frac{5}{3} \tilde{r}\right) j_{3}(q R)\right)
$$

Here $-Z e$ is the nuclear charge, $Q_{0}=\sqrt{\frac{3}{5 \pi}} R^{2} \beta$ is the static quadrupole moment and $R=r_{0} A^{1 / 3}$. One thus observe that the longitudinal multipoles are insensitive to the rotational components of the velocity field, since their value is constant for any value of the rigidity parameter $\tilde{r}$. Moreover this value is proportional to the transverse multipole for irrotational flow

$$
\hat{T}_{2 \mu}^{L}(q, \tilde{r})=\sqrt{\frac{2}{3}} \hat{T}_{2 \mu}^{E}(q, \tilde{r}=0)
$$

The next step consists in the study of low momentum transfer behaviour of the above discussed multipoles. One obtain the well known Siegert theorem which states that in the long-wavelength limit the transverse electric multipole can be expressed in terms of the longitudinal one

$$
\hat{T}_{2 \mu}^{E}(q \approx 0, \tilde{r})=\sqrt{\frac{3}{2}} \hat{T}_{2 \mu}^{L}(q \approx 0, \tilde{r})
$$

Recalling the remark that we have made above about the constancy of the longitudinal multipole with $\tilde{r}$, one conclude that the proportionality between this multipole and the 
transverse electric one is satisfied for the IF for arbitrary momentum transfer $q$. For the $\mathrm{RR}$ this relation is valid only for low- $q$ transfer. This means that in principle the reactions performed at low- $q$ are not able to give informations about the vorticity. The Riemann rotator behaves as an irrotational liquid drop at small transferred momentum. From the point of view of the multipole expansion this fact can be understood invoking the following argument : in the low- $q$ limit the longitudinal multipole is proportional to the time derivative of the charge quadrupole moment operator

$$
\hat{T}_{2 \mu}^{L}(q \approx 0, \tilde{r})=-\frac{i q}{15} \dot{\hat{Q}}_{2 \mu}
$$

where the charge quadrupole moment has the well-known form

$$
\hat{Q}_{\lambda \mu}=\int d^{3} r r^{\lambda} Y_{\lambda \mu} \hat{\rho}(\boldsymbol{r}, t)
$$

According to the charge-current conservation law

$$
\frac{\partial \hat{\rho}}{\partial t}+\nabla \cdot \hat{\boldsymbol{J}}=0
$$

the time derivative of the charge quadrupole $\dot{\hat{Q}}_{2 \mu}$ may be put in the form

$$
\dot{\hat{Q}}_{2 \mu}=\int d^{3} r r^{\lambda} Y_{\lambda \mu} \nabla \cdot \hat{\boldsymbol{J}}
$$

Since the gradient cancels the rotational (vortical) components of the nuclear current, the time derivative of the charge quadrupole moment is to be associated with curless quadrupole flows, i.e. $\boldsymbol{\nabla} \times \hat{\boldsymbol{J}}=0$, like the $\beta$ and $\gamma$ vibrations. This is the reason why the nuclear response is vibrating-like (without shear components) for low- $q$ transfer. Therefore in order to obtain informations about the rotational currents inside the nucleus one must go beyond the Siegert theorem by increasing the energy transferred in the scattering reaction. The high- $q$ terms arising in the multipole expansion of the transverse electric multipole are free of the charge-current conservation constraint. Hence they could provide us with information about the vortical components, i.e. $\boldsymbol{\nabla} \times \hat{\boldsymbol{J}} \neq 0$. In order to accomplish this task we employ the above mentioned multipole expansion formalism for the transverse electric multipoles, where the Siegert limit term is explicitly decoupled from the high- $q$ terms. Equation (7) is rewritten in the quadrupole case as follows by factorizing the longitudinal multipole in the $q=0$ point

$$
t_{E 2}(q, \tilde{r})_{f i}=\sqrt{\frac{3}{2}} t_{L 2}(0)_{f i}\left(1-\eta_{2}(q, \tilde{r})\right)
$$

In the above equation we have introduced a quantity related to the deviation from the Siegert theorem that is also given as a convex combination of rigid and irrotational values

$$
\eta_{2}(q, \tilde{r}) \equiv \frac{q^{2} t_{T 2}(q, \tilde{r})_{f i}}{t_{E 2}(0, \tilde{r})_{f i}}=\tilde{r} \eta_{2}(q, \tilde{r}=1)+(1-\tilde{r}) \eta_{2}(q, \tilde{r}=0)
$$


In Fig.1 a plot of this quantity vs. momentum transfer is presented. The RR model displays a stronger deviation from the Siegert theorem than the IF model. For the hexadecupole case $(\lambda=4)$, the effect is lowered with respect to the quadrupole case $(\lambda=2)$ for both models. Another quantity of interest defined in electron scattering theory [19] is the real transverse electric form factor

$$
F_{\lambda}^{E}(q) \equiv \frac{t_{E \lambda}(q)}{t_{E \lambda}(0)}
$$

with $F_{\lambda}^{E}(0)=1$. Specializing to the quadrupole case of the Riemann Rotator, expanding this form factor according to the Dubovik-Cheshkov procedure and using the definition of the toroidal multipole moment [eqs.(9) and (19)] one gets in the first order of $q^{2}$

$$
F_{2}^{E}(q) \approx 1-\frac{q^{2}}{3} \mathcal{I}_{\tilde{r}} \cdot \frac{\mathcal{T}_{2}}{Q_{2}}
$$

where $\mathcal{I}_{\tilde{r}}$ is the inertia moment of the Riemann Rotator and $Q_{2}=3 e^{2} Z R_{0}^{2} \beta / 4 \pi$ is the r.m.e. of the transition charge quadrupole moment, which does not depend on the rigidity parameter $\tilde{r}$. Recalling the fact that at small values of $q$ the slope of the real

Table 1. Toroidal quadrupole transition moment $\mathcal{T}_{2}$ in arbitrary units at fixed deformation $\beta$ for ${ }^{152} \mathrm{Sm},{ }^{154} \mathrm{Sm}$ and ${ }^{166} \mathrm{Er}$ for three different values of the rigidity parameter $\tilde{r}$ : IF $(\tilde{r}=0)$, RR $(\tilde{r}=1)$ and for the Riemann Rotational Model with $\tilde{r}=0.5$.

\begin{tabular}{lllll}
\hline Nucleus & & & \multicolumn{1}{r}{} & \multicolumn{1}{c}{$\mathcal{T}_{2}$} \\
\hline \multirow{2}{*}{${ }^{152} \mathrm{Sm}$} & 0.246 & 0. & $5.2 \cdot 10^{-4}$ \\
& & 0.5 & $1.32 \cdot 10^{-3}$ \\
& & 1. & $1.72 \cdot 10^{-2}$ \\
\hline \multirow{2}{*}{${ }^{154} \mathrm{Sm}$} & 0.270 & 0. & $5.6 \cdot 10^{-4}$ \\
& & 0.5 & $1.42 \cdot 10^{-3}$ \\
& & 1. & $1.56 \cdot 10^{-2}$ \\
\hline \multirow{2}{*}{${ }^{166} \mathrm{Er}$} & 0.287 & 0. & $5.2 \cdot 10^{-4}$ \\
& & 0.5 & $1.59 \cdot 10^{-3}$ \\
& & 1. & $1.52 \cdot 10^{-2}$ \\
\hline
\end{tabular}

Coulomb (magnetic) form-factor allow us to determine the root-mean-square charge (magnetic) radii [19], we proceed in the same fashion to seek the transition toroidal quadrupole moment. According to eq.(23) and the definition of the real transverse electric form-factor (25) the toroidal quadrupole moment, for an arbitrary value of the rigidity parameter, reads

$$
\mathcal{T}_{2}(\tilde{r})=\frac{3 e^{2} Z}{56 \pi} \frac{(3+2 \tilde{r})}{\mathcal{I}_{\tilde{r}}} R_{0}^{4} \beta
$$


Values of this transition moment for different nuclei and rigidity parameters are given in table 1 . We have also plotted $\mathcal{T}_{2}$ versus the rigidity parameter $\tilde{r}$ for ${ }^{152} \mathrm{Sm}$ and ${ }^{166} \mathrm{Er}$ (fig.2). An obvious, but important conclusion in our view represents the fact that the toroidal quadrupole moment drastically increase for nuclei which exhibite vortical components of the flow, being two order in magnitude greater in the $\mathrm{RR}$ case relative to the IF case.

\section{Observation of toroidal quadrupole transitions in electron scattering}

In view of the definition of toroidal multipoles their measurement is equivalent to the measurement of the transverse electric multipole at high-momentum transfer and afterwards the substraction of the Siegert limit. The use of inelastic electron scattering allows the measurement of form factors (f.f.) at momentum transfers ranging from the photon point to $q R \gg 1$. Therefore it is possible to get information about the transverse f.f. for $q$ values which make the charge-current constraints important up to $q$-regions where the Siegert theorem breaks up and the toroidal f.f. dominates the transverse electric part of the cross-section. The cross-section for inelastic electron scattering on an unpolarized nucleus can be written in the PWBA as [20]

$$
\begin{aligned}
\frac{d \sigma}{d \Omega}=4 \pi \sigma_{M o t t} & \frac{1}{2 I_{i}+1}\left\{\frac{q_{\mu}^{4}}{q^{4}} \sum_{\lambda=0}^{\infty}\left|\left\langle I_{f}\left\|\hat{M}_{\lambda}(q)\right\| I_{i}\right\rangle\right|^{2}\right. \\
& \left.+\left(\frac{q_{\mu}^{2}}{2 q^{2}}+\tan ^{2} \frac{\theta}{2}\right) \sum_{\lambda=1}^{\infty}\left|\left\langle I_{f}\left\|\hat{T}_{\lambda}^{E}(q)\right\| I_{i}\right\rangle\right|^{2}+\left|\left\langle I_{f}\left\|\hat{T}_{\lambda}^{M}(q)\right\| I_{i}\right\rangle\right|^{2}\right\}
\end{aligned}
$$

where $\sigma_{M o t t}$ is the Mott cross section, $q_{\mu}=(\boldsymbol{q}, \omega)=P_{i \mu}-P_{f \mu}$ is the four momentum transfer from the nucleus, $P_{i \mu}$ and $P_{f \mu}$ are the initial and final nuclear four-momenta, $q=|\boldsymbol{q}|, q_{\mu}^{2}=q^{2}-\omega^{2}$ and $\hat{M}_{\lambda}, \hat{T}_{\lambda}^{E}$ and $\hat{T}_{\lambda}^{M}$ are the Coulomb, transverse electric and magnetic multipoles respectively. In the case of exciting the g.s. band of an even-even nucleus there will be involved the electric multipoles with $\lambda \geq 2$ and the magnetic multipoles with $\lambda$-odd. In the present work for simplicity we shall discard the magnetic multipoles, thereby confining ourselves to the analysis of longitudinal and transverse electric parts of the cross section. For our present purposes, which consists in the determination of toroidal transitions in connexion with the possibility of observing nuclear vortical currents we must chose an appropriate method to subtract the dominant longitudinal component from the cross section and therefore obtaining the searched transverse multipoles. The method of separating the transverse multipole at $180^{\circ}$ angle scattering is very seducing, since at such angles they are dominating the cross-section. During the last times there have been reported measurements of normal-parity transverse excitations in such experiments [21]. Another method of separating the transverse multipole from the longitudinal one consists in performing 
scattering of polarized electrons on polarized nuclear targets. Such processes allow the measurement of observables which involve the interferences between longitudinal and transverse multipoles [3,22]. In this paper we use the first method because it does not imply the complex calculations required by the second method.

For the excitation $0^{+} \rightarrow \lambda^{+}$it turns out the following expression for the differential cross-section

$$
\frac{d \sigma}{d \Omega}=4 \pi \sigma_{M o t t} f_{r e c}^{-1}\left\{\frac{q_{\mu}^{4}}{q^{4}}\left|t_{C \lambda}(q)_{0^{+} \rightarrow \lambda^{+}}\right|^{2}+\left(\frac{q_{\mu}^{2}}{2 q^{2}}+\tan ^{2} \frac{\theta}{2}\right)\left|t_{E \lambda}(q)_{0^{+} \rightarrow \lambda^{+}}\right|^{2}\right\}
$$

Using the multipole decomposition which separates the toroidal multipoles and neglecting in the above expression of the differential cross-section the Coulomb multipoles and the low- $q$ limit of the transverse electric multipole one get

$$
\frac{d \sigma}{d \Omega}=4 \pi \sigma_{M o t t} f_{r e c}^{-1} q^{2}\left(\frac{1}{2} q_{\mu}^{2}+q^{2} \tan ^{2} \frac{\theta}{2}\right)\left|t_{T \lambda}(q)_{0^{+} \rightarrow \lambda^{+}}\right|^{2}
$$

This approximation is equivalent to neglecting the transition charge moments $Q_{\lambda}$ and the average 2 n-power radii of the charge distribution $r_{\lambda}^{2 n}$ defined in $[4,14,19]$. We have represented graphically the differential cross-sections for the RR and IF models in both cases, the exact (29) and approximate (30), for the quadrupole (fig.3a) and hexadecupole (fig.3b) transitions induced by the scattered electron. It is transparent from these figures that the quoted approximation, works well for the RR model at low and high momentum transfer for both considered transitions. The principal difference appears in the location of the diffraction minima which are lowered in the approximate case relative to the exact one. However this is not very important since in a phase shift analysis the curve in the neighborhood of the sharp minimas is smoothed. In the IF case the agreement between the exact and approximate differential cross-section is good for $q<400 \mathrm{MeV} / c$ for $\lambda=2$ and $q<250 \mathrm{MeV} / c$ for $\lambda=4$. The reason which determines the discrepancies for the IF model between the the exact and approximate curves steems on the fact that at higher momentum transfer the 2n-power radii of the charge multipoles contribution in the cross-section is enhanced.

\section{Conclusions}

We end-up this paper by substantiating the main results:

(i) The quantity called deviation from the Siegert theorem introduced in this paper, which describes the contribution of high- $q$ terms in the expression of the transverse electric multipole contains the information about the currents inside the nucleus. For the RR which is a submodel of the more general Riemann model, the deviation from the Siegert limit has a larger slope than the corresponding quantity defined for the IF model. The deviation from the Siegert theorem increase with the 
rigidity parameter $\tilde{r}$ and the mass number $\mathrm{A}$ for the considered nuclei in the range $q=0 \div 150 \mathrm{MeV} / c$ and oscillates around $\eta=1$ above $q=150 \mathrm{MeV} / c$.

(ii) The transition toroidal quadrupole moment is a monotous increasing function of the vorticity. This strengths our opinion that the toroidal moments are to be associated with vortical electromagnetic currents in the same way as the charge multipole moments are associated with charge distribution or irrotational electromagnetic currents.

(iii) At backward electron scattering the differential cross section of the RR model may be approximated by taking into account only the toroidal form factor for a broad range on momentum transfer.

\section{Acknowledgments}

I would like to express my gratitude to A.A. Răduţă for reading the manuscript and his interesting remarks. I am also expressing my thanks for valuable discussions with V.M. Dubovik, P.G.H. Sandars, J.D. Walecka, E. Moya de Guerra, N. Lo Judice and R. Liotta. The assistance of A.Andronic is acknowledged.

\section{References}

[1] Rowe D J 1970 Nuclear Collective Motion (London:Methuen)

[2] Rosensteel G 1988 Ann. Phys., NY 186230

Rosensteel G 1990 Phys. Rev. C41 R811

Rosensteel G 1992 Phys. Rev. C46 1818

[3] Moya de Guerra E 1986 Phys. Rep. 138293

[4] Dubovik V M and Cheshkov 1974 Fiz.El.Chast.At.Yad. 5791

[5] Zel'dovich Ya B 1957 Sov.Phys.-JETP 61184

[6] Dubovik V M and Cheshkov A A 1967 Sov.Phys.-JETP 4111

[7] Moskalev A N and Porsev S G 1989 Yad.Fiz. 491266

[8] Sandars P G H 1993 Phys. Scr. T46 16

[9] Balbutsev E B and Mikhailov I N 1988 J. Phys. G: Nucl. Part. Phys. 14545

[10] Bastrukov S I, Mişicu Ş and Sushkov A V 1993 Nucl. Phys. A562 191

[11] Mişicu Ş 1994 in Frontier Topics in Nuclear Physics, Predeal, Romania (New York:Plenum Press)

[12] Edmonds A R 1960 Angular Momentum in Quantum Mechanics (Princeton:PUP)

[13] Siegert A J F 1937 Phys. Rev. 52787

[14] Dubovik V M and Tugushev V V 1990 Phys. Rep. 187145

[15] Blatt J M and Weisskopf V F 1952 Theoretical Nuclear Physics (New York:Willey)

[16] Friar J L and Fallieros S 1984 Phys. Rev. C29 1645

[17] Gulshani P and Rowe D J 1976 Can. J. Phys. 54970

[18] Weaver L, Cusson R Y and Bidenharn L C 1976 Ann. Phys., NY 102493

[19] Überall H 1971 Electron Scattering from Complex Nuclei (New York:Academic Press) 
[20] de Forest T Jr and Walecka J D 1966 Adv. Phys. 151

[21] Richter A 1994 in Proc. of the V La Rabida Int. Summer School on Response of the Nuclear System to External Forces, La Rabida, Spain (to be published)

[22] Donnelly T W , Raskin A S and Dubach J 1987 Nucl. Phys. A474 307 


\section{Figure captions}

Figure 1. The deviation from the Siegert theorem for the the nucleus ${ }^{166} \mathrm{Er}$ for the excitations $0^{+} \rightarrow 2^{+}(\lambda=2)$ and $0^{+} \rightarrow 4^{+}(\lambda=4)$ considering the case of IF and RR.

Figure 2. Transition toroidal quadrupole moment dependence on the rigidity parameter for ${ }^{152} \mathrm{Sm}(-)$ and ${ }^{166} \operatorname{Er}(---)$

Figure 3. Differential cross section versus momentum transfer for (a) $\lambda=2$ and (b) $\lambda=4$ for both IF and RR. For each model two sets of datta are ploted. One is obtained by using eq.(29) which includes the Coulomb ans transverse electric form-factors the other use only the toroidal form factor (eq.(30)). 